\title{
Characterization of exopolysaccharides produced by Bifidobacterium longum NB667 and its cholate-resistant derivative strain IPLA B667dCo
}

\begin{tabular}{|r|l|}
\hline Journal: & Journal of Agricultural and Food Chemistry \\
\hline Manuscript ID: & jf-2011-04034n.R2 \\
\hline Manuscript Type: & Article \\
\hline Author: & 27-Dec-2011 \\
\hline & $\begin{array}{l}\text { Salazar, Nuria; Instituto de Productos Lacteos de Asturias, Consejo } \\
\text { Superior de Investigaciones Científicas, Microbiology and } \\
\text { Biochemistry of Dairy Products } \\
\text { Ruas-Madiedo, Patricia; Instituto de Productos Lacteos de Asturias, } \\
\text { Consejo Superior de Investigaciones Científicas, Microbiology and } \\
\text { Biochemistry of Dairy Products } \\
\text { Prieto, Alicia; Centro de Investigaciones Biologicas, Consejo Superior } \\
\text { de Investigaciones Científicas (CIB-CSIC), } \\
\text { Calle, Luis; Centro de Investigaciones Biológicas, CSIC, Protein } \\
\text { Science } \\
\text { de los Reyes-Gavilan, Clara; Instituto de Productos Lacteos de } \\
\text { Asturias, Consejo Superior de Investigaciones Científicas, } \\
\text { Microbiology and Biochemistry of Dairy Products }\end{array}$ \\
\hline
\end{tabular}

\section{SCHOLARONE" Manuscripts}


1 Title: Characterization of exopolysaccharides produced by Bifidobacterium longum

$2 \quad$ NB667 and its cholate-resistant derivative strain IPLA B667dCo

3

4 Authors: Nuria Salazar ${ }^{1}$, Patricia Ruas-Madiedo ${ }^{1}$, Alicia Prieto ${ }^{2}$, Luis P. Calle ${ }^{2}$, and

5 Clara G. de los Reyes-Gavilán ${ }^{1^{*}}$

6

7 Addresses:

8 1. Instituto de Productos Lácteos de Asturias-Consejo Superior de Investigaciones

9 Científicas (IPLA-CSIC), Departamento de Microbiología y Bioquímica de

10 Productos Lácteos. Carretera de Infiesto s/n, 33300 Villaviciosa, Asturias, Spain.

11

12 2. Centro de Investigaciones Biológicas (CIB-CSIC), Ramiro de Maeztu 9, 28040 13 Madrid, Spain.

18 *Clara G. de los Reyes-Gavilán (Tel: 34 985893335; Fax: 34 985892233; E-mail:

19 greyes_gavilan@ipla.csic.es) 
21 ABSTRACT

Bifidobacteria are natural members of the human intestinal microbiota and some strains are being used as probiotics. Adaptation to bile can allow them to increase survival in gastrointestinal conditions, thus improving their viability. Bifidobacterium longum NB667 and the cholate-resistant strain B. longum IPLA B667dCo produced exopolysaccharides (EPS) that were partially characterized. Analysis by size exclusion chromatography-multiangle laser light scattering indicated that the EPS crude fractions of both strains contained two polymer peaks of different molar mass. Based on chromatographic techniques both peaks appeared to be heteropolysaccharides. The smaller peak was mainly composed of glucose, galactose and rhamnose whose molar ratios and linkage types showed slight variations between the EPS fractions of both strains. The bigger peak consisted of glucose and galactose; the monosaccharide composition was identical in the EPS fractions of the two microorganisms, but their

34 infrared spectra presented some differences regarding compounds other than carbohydrates that seem to be associated to the polymer. Differences in the composition of EPS fractions did not affect the capability of crude EPS from B. longum to be

37 fermented by the human intestinal microbiota in fecal batch cultures. 


\section{INTRODUCTION}

Bifidobacteria are natural members of the human intestinal microbiota, in which

47 they occur at concentrations of $10^{9}$ to $10^{11}$ cells per gram of feces, representing up to $91 \%$ of the total gut population in the early stages of life. ${ }^{1}$ Great variability regarding microbiota composition and bifidobacteria levels can be found among different individuals, which could be affected by food intake, age, and individual characteristics, among other factors. Some health-promoting properties have been attributed to bifidobacteria, which are being included in a huge variety of fermented milks and functional foods. ${ }^{2}$ Once ingested with food, probiotics must overcome biological barriers present in the gastrointestinal tract (GIT), mainly acid in the stomach and bile in the intestine. Several strategies are currently available to improve bifidobacterial survival, one of the more suitable being the pre-exposure to sublethal stresses or the selection of derivative strains by stress adaptation. ${ }^{3}$ In this way, bifidobacteria can develop a stable bile-resistant phenotype; this adaptation process has been related to cross-resistance to other stressing factors as well as with other pleiotropic changes in cells. $^{4-7}$

Many strains of lactic acid bacteria (LAB) and bifidobacteria are able to produce exopolysaccharides (EPS). Some EPS-producing strains are being used in the dairy

63 industry because of their suitable technological properties. ${ }^{8,9}$ However, the 64 physiological functions of these biopolymers have not been clearly determined yet. ${ }^{10}$

65 Among the beneficial effects attributed to EPS are a cholesterol-lowering ability, an 66 immunomodulating capability, and the possibility of acting as prebiotics. Regarding 67 their structure and composition, EPS from LAB are generally divided into 68 homopolysaccharides (HoPS), which are polymers composed of one type of 69 monosaccharide, and heteropolysaccharides (HePS), which are polymers of repeating 
70 units that are composed of two or more types of monosaccharides. Studies on the

71 physical properties, composition and structure of polymers produced by bifidobacteria

72 are currently scarce. ${ }^{10}$ We have recently found that some human intestinal

73 Bifidobacterium isolates were able to produce $\mathrm{HePS}^{11}$ that served as fermentable

74 substrates for the human intestinal microbiota. ${ }^{12}$ In addition, bile exposure is able to

75 trigger the synthesis of EPS in Bifidobacterium animalis. ${ }^{13}$ Acquisition of a bile-

76 resistant phenotype has been recently associated with changes in the EPS fractions of

77 some $\mathrm{LAB}$ and Bifidobacterium species of non-human origin. In this respect, the

78 spontaneous acquisition of a "ropy" phenotype in a bile-resistant derivative of $B$.

79 animalis was correlated with variations in molar mass (MM) and ratios among the

80 monosaccharides glucose, galactose and rhamnose, which are components of the EPS

81 fractions synthesized by original and bile-adapted strains. ${ }^{14}$ In Lactobacillus delbrueckii

82 subsp. lactis, the acquisition of a bile-resistant phenotype promoted slight changes in

83 glycosidic linkages of the EPS which affected some technological and functional

84 properties of the producing microorganism. ${ }^{15}$

85 Taking into consideration the data commented on above, the aim of the present

86 work was to gain insight into the influence that adaptation to bile could exert on some

87 physico-chemical and biological characteristics of EPS produced by the species

88 Bifidobacterium longum, a normal inhabitant of the human GIT. The strain B. longum

89 NB667 and its corresponding cholate-resistant derivative B. longum IPLA B667dCo

90 were used as the model of study.

91

92 MATERIAL AND METHODS

93 EPS-producing strains and culture conditions 
Food Research Collection (Ede, the Netherlands) was originally isolated from infant feces. B. longum IPLA B667dCo is a cholate-adapted strain, belonging to the IPLA maintained at $-80{ }^{\circ} \mathrm{C}$ in MRSC broth [MRS broth (Biokar Diagnostics, Beauvais, France) plus $0.25 \%(w / v)$ L-cysteine (Sigma-Adrich Chemical Co., St. Louis, MO,

100 USA)] with $20 \%$ glycerol. For the isolation of EPS, frozen stocks were grown overnight in MRSC at $37{ }^{\circ} \mathrm{C}$ in an anaerobic cabinet (Mac 500; Don Whitley Scientific, West Yorkshire, UK) under a $10 \% \mathrm{H}_{2}, 10 \% \mathrm{CO}_{2}$, and $80 \% \mathrm{~N}_{2}$ atmosphere. Cultures $(200 \mu \mathrm{L})$ were used to inoculate agar-MRSC plates by means of sterile glass beads. Plates were incubated for 5 days at $37^{\circ} \mathrm{C}$ in anaerobic conditions.

\section{EPS isolation}

Crude EPS produced by Bifidobacterium strains were purified from the cellular biomass harvested from MRSC agar plates. ${ }^{16}$ Biomass was collected using $2 \mathrm{~mL}$

109 ultrapure water per plate and the resulting volume mixed with 1 volume of $2 \mathrm{M} \mathrm{NaOH}$.

110 The suspension was gently stirred overnight at room temperature to promote polymer 111 release from the cellular surface. Cells were removed by centrifugation at $8,400 \times g$ for

$11230 \mathrm{~min}$ and EPS was precipitated from the supernatants over $48 \mathrm{~h}$ at $4{ }^{\circ} \mathrm{C}$ using 2

113 volumes of cold absolute ethanol. Following centrifugation, the EPS fraction was

114 resuspended in ultrapure water and dialyzed for 3 days at $4{ }^{\circ} \mathrm{C}$ with daily changes of 115 water, using dialysis tubes (Sigma) of 12 to $14 \mathrm{kDa}$ molecular mass cut off. Finally, the 116 dialyzed crude EPS preparations were freeze-dried. 
For analytical purposes, the weight average MM (Mw) distribution of the crude

120 EPS was measured by SEC and multi-angle laser light scattering detection (SEC-

121 MALLS) as described by Salazar et al. ${ }^{17}$ Diffusion ordered NMR spectroscopy (DOSY)

122 was also used as an additional procedure to ascertain the Mw of these polymers through

123 the measurement of diffusion coefficients ${ }^{18}$ as described by Leal et al. ${ }^{19}$ The protein

124 content of the polymers was determined by the BCA protein assay kit (Pierce,

125 Rockford, IL, USA) following the manufacturer's instructions.

126 For EPS fractionation preparative SEC was used: $20 \mathrm{mg}$ of crude EPS were

127 dissolved in $2 \mathrm{~mL}$ of $0.3 \mathrm{M} \mathrm{NaOH}$ and centrifuged at $16,000 \times \mathrm{g}$ for $15 \mathrm{~min}$ in an

128 Eppendorf 5415D microcentrifuge to eliminate insoluble material. The supernatant was

129 then subjected to SEC in a Sepharose CL6B (GE Healthcare, Madrid, Spain) column

$130(60 \times 2.6 \mathrm{~cm})$ equilibrated with $0.3 \mathrm{M} \mathrm{NaOH}$ at a flow rate of $18 \mathrm{~mL} \mathrm{~h}^{-1}$. EPS fractions

131 (3.5 mL) were recovered and monitored for carbohydrates using the phenol-sulfuric acid

132 method. $^{20}$ Apparent average MM of EPS eluted peaks was obtained using a calibration

133 curve made with commercial standards (Dextran Blue, Dextrans T70, and T10

134 [Pharmacia, Uppsala, Sweden], and vitamin B12 [Merck, Darmdstadt, Germany]). The

135 positive fractions for carbohydrates were appropriately combined, dialyzed against water,

136 concentrated to a small volume, and freeze-dried. The amount of each purified fraction

137 was determined gravimetrically.

138

139 Chemical characterization of EPS fractions

140 Monosaccharide composition and phosphate content

141 For analysis of neutral sugars in the two crude EPS and the SEC-fractionated

142 EPS peaks, polysaccharides were hydrolyzed with $1.5 \mathrm{M}$ trifluoraoacetic acid (TFA) for

$1431 \mathrm{~h}$ at $121^{\circ} \mathrm{C}$. The resulting monosaccharides were converted into their corresponding 
144 alditol acetates, ${ }^{21}$ which were identified and quantified by gas-liquid chromatography 145 (GLC) on a 7980A instrument (Agilent Technologies Inc., Palo Alto, CA) equipped 146 with a flame ionization detector, using an Agilent HP5 fused silica column (30 m x 0.25

$147 \mathrm{~mm}$ internal diameter $\mathrm{x} 0.2 \mu \mathrm{m}$ film thickness) and the following temperature program: $148160{ }^{\circ} \mathrm{C}$ for $5 \mathrm{~min}$, then $3.5^{\circ} \mathrm{C} \min ^{-1}$ to $205^{\circ} \mathrm{C}$ and finally $210{ }^{\circ} \mathrm{C}$ for $0.5 \mathrm{~min}$. Phosphate 149 content was deduced from inorganic phosphate determination on a 5500 Inductively 150 Coupled Plasma instrument (Perkin Elmer, San Jose, CA, USA).

\section{Monosaccharide linkage types}

The linkage types present in the EPS molecules were determined after methylation of the SEC-fractioned EPS according to the procedure described by

155 Ciucanu and Kerek. ${ }^{22}$ The permethylated polysaccharide was hydrolyzed with $3 \mathrm{M}$

156 TFA, and the released monosaccharides were reduced with $\mathrm{NaBD}_{4}$ and then acetylated 157 to give their corresponding partially methylated alditol acetates, which were analyzed by GLC and mass spectrometry (GLC-MS) under conditions previously described. ${ }^{23}$

Fourier-transform infrared spectroscopy (FT-IR)

Fourier transform (FT) Infrared (IR) spectra were obtained by the $\mathrm{KBr}$

162 technique ${ }^{24}$ using a FTIR 4200 type A instrument (Jasco Corporation, Tokyo, Japan).

163 Transmittance was measured in a spectral range of $400-4000 \mathrm{~cm}^{-1}$. A Deuterated L-

164 Alanine Triglycine Sulphate (TGS) detector was used with $4 \mathrm{~cm}^{-1}$ data resolution.

\section{Fermentation of bacterial EPS in fecal cultures}

\section{$167 \quad$ Fecal batch cultures}

168 Three independent fecal batch fermentations, each of them corresponding to 
169 samples of three different healthy adult donors (2 women and 1 man, 25 to 37 years

170 old), were carried out in the carbohydrate-free basal medium (CFBM) previously

171 described. ${ }^{12}$ For each batch, CFBM was distributed into different glass tubes that were

172 added with the crude EPS isolated from each of the two Bifidobacterium strains. One

173 additional tube was kept without adding carbon source and was used as a control. The

174 fecal batch cultures were performed in the conditions indicated previously. ${ }^{12}$ Samples

175 for analyses were taken from fecal cultures at times 0,1 , and 5 days. Eight hundred $\mu \mathrm{L}$

176 of cultures were centrifuged $(12000 \times \mathrm{g}, 10 \mathrm{~min})$ each time, and pellets and supernatants

177 were collected.

178

\section{Analysis of Short Chain Fatty Acids (SCFA) by GC-MS}

180

Cell-free supernatants from fecal batch cultures were filtered through $0.2 \mu \mathrm{m}$

181 filters and mixed with $1 / 10$ of ethyl-butyric $\left(2 \mathrm{mg} \mathrm{mL}^{-1}\right)$ as an internal standard and 182 stored at $-20^{\circ} \mathrm{C}$ until analysis.

183 A system composed of a gas chromatograph (GC) 6890N (Agilent) connected 184 to a MS 5973N detector (Agilent) was used to quantify the SCFA. Data were collected 185 with the Enhanced ChemStation G1701DA software (Agilent). Samples (1 $\mu \mathrm{L})$ were 186 analyzed as previously described by Salazar and co-workers. ${ }^{12}$ Total SCFA 187 concentrations were calculated as the sum of the three major SCFA (acetic + propionic $188+$ butyric). The molar proportion of each SCFA was obtained as the concentration 189 percentage with respect to the total SCFA.

191 Analysis of bifidobacteria by quantitative real-time PCR (qPCR)

192 The quantification of the Bifidobacterium population in fecal batch cultures 193 was performed by qPCR using previously described genus-specific primers. ${ }^{25}$ DNA was 
194 extracted from pellets harvested from $800 \mu \mathrm{L}$ of fecal batch cultures. Cells were washed 195 once in PBS buffer and DNA was extracted with the QIAamp® DNA Stool Kit (Qiagen $196 \mathrm{GmbH}$, Hilden Germany) following the manufacturer's instructions. Purified DNA 197 samples were stored at $-20^{\circ} \mathrm{C}$ until use.

All reactions were performed on MicroAmp optical plates sealed with MicroAmp optical caps (Applied Biosystems, Foster City, CA) and amplifications were carried out in a 7500 Fast Real Time PCR System (Applied Biosystems) using the SYBR Green PCR Master Mix (Applied Biosystems). One $\mu \mathrm{L}$ of purified DNA was used as the template in the $25 \mu \mathrm{L}$ PCR reaction. Thermal cycling consisted of an initial cycle of $95^{\circ} \mathrm{C}$ for $10 \mathrm{~min}$ followed by 40 cycles of $95^{\circ} \mathrm{C}$ for $15 \mathrm{~s}$ and $60{ }^{\circ} \mathrm{C}$ for $1 \mathrm{~min}$. Standard curves were made with the strain B. longum NCIMB8809 which was grown overnight in MRSC under anaerobic conditions. Standard curves were obtained by plotting the $C_{t}$ values obtained for the standard culture as a linear function of the base10 logarithm of the initial number of cells in the culture determined by plate counting. The number of Bifidobacterium cells in fecal samples was determined by comparing the $\mathrm{C}_{\mathrm{t}}$ values obtained to the standard curve. The detection limit with primers and qPCR conditions used was $5 \times 10^{4}$ cells $\mathrm{g}^{-1}$. Samples were analyzed in duplicate in at least two

211 independent PCR runs.

\section{Statistical analysis}

The statistical analysis of SCFA data was performed with the SPSS-PC 15.0 software package (SPSS Inc., Chicago, IL, USA). Independent one-way ANOVA tests

216 were performed at 1 and 5 days of incubation. Differences among culture conditions

217 (control, and EPS NB667 and IPLA B667dCo as carbon sources) were assessed by means of the LSD (least significant difference) mean comparison test $(\mathrm{p}<0.05)$. 


\section{RESULTS AND DISCUSSION}

Physico-chemical characterization of the EPS from the parental and the cholateresistant derivative strains

Crude EPS polymer preparations were isolated from strains grown on MRSC plates instead of a complex liquid medium in order to avoid contamination with glucomannans present in the yeast extract added to MRS medium, as previously indicated by Salazar et al. ${ }^{12}$ The level of EPS production by B. longum NB667 and $B$. longum IPLA B667dCo was about $180-190 \mathrm{mg}$ polymer per 50 plates in both microorganisms, similar to that reported previously for the parental strain NB667. ${ }^{11}$ The content of protein in both crude EPS ranged between 2.2 and $2.7 \%$, these values being

231 in the same order as those previously reported by Salazar et al. ${ }^{12}$ for EPS synthesized by bifidobacteria from intestinal origin.

With the aim of knowing the physico-chemical characteristics of the EPS synthesized by B. longum and the possible influence that the acquisition of resistance to cholate could exert on them, EPS crude preparations from the two microorganisms under study were first compared. Analysis by SEC confirmed that the EPS crude fractions of

237 both strains contained two polymer peaks of low (around $4 \times 10^{3} \mathrm{~g} \mathrm{~mol}^{-1}$ ) and high (around

2384 to $5 \times 10^{6} \mathrm{~g} \mathrm{~mol}^{-1}$ ) apparent average MM (Table 1), as was recently reported for the 239 parental strain B. longum NB667. ${ }^{14}$ In EPS NB667 both peaks were present in similar 240 amounts, whereas in EPS IPLA B667dCo the biggest peak was slightly less abundant than 241 the smaller one. The presence of two peaks of different MM seems to be a relatively

242 common feature in EPS fractions isolated from bifidobacteria. ${ }^{1426-28}$ Analysis by SEC243 MALLS of crude EPS preparations revealed clear differences on the physico-chemical 
244 parameters ( $\mathrm{Mw}, \mathrm{Rg}$, and $v$ ) between peaks of low and high MM in both strains (Table 1).

245 In addition, a significant amount of protein appeared associated with the peak of low MM 246 (peak 2) in the two strains under study (Figure 1). Remarkably, Mw of peak 1 from EPS 247 NB667 and IPLA B667dCo estimated by SEC-MALLS dropped dramatically with respect 248 to the apparent MM calculated by retention times in SEC (Table 1) but were of the same 249 order of magnitude as those obtained by DOSY $\left(2.2 \times 10^{5} \mathrm{~g} \mathrm{~mol}^{-1}\right.$ and $5.5 \times 10^{5} \mathrm{~g} \mathrm{~mol}^{-1}$ for 250 peak 1 of EPS NB667 and IPLA B667dCo, respectively). Reasons for these discrepancies will be discussed later. Rhamnose, galactose and glucose were released after acid hydrolysis of the crude EPS preparations of both strains and minor amounts of mannose were also obtained; the monosaccharide relative ratios displayed minor differences between the parental strain and the cholate-resistant derivative (Table 1). Monosaccharide composition of both EPS crude polymers was similar to that found in other LAB and bifidobacteria from food and intestinal origin. ${ }^{27,} 29$ The presence of significant amounts of uronic acids or amino sugars was ruled out in crude EPS preparations of both strains based on the lack of characteristic absorption bands in the IR-FT spectra (data not shown). Phosphate determinations rendered only trace amounts (0.6-1.0\%).

260 Therefore, in a step forward crude EPS were submitted to preparative SEC in order 261 to separate the two fractions of low and high MM for accomplishing further 262 characterization of these two polymers. The separation of both fractions was complete

263 (Figure 2); however, the monosaccharide recovery after acid hydrolysis of the bigger MM 264 peak was low (about 40\%) whereas that of the smaller peak was close to $80 \%$. Other 265 authors have also reported low yield of recovered monosaccharides from polymer fractions 266 in the genus Bifidobacterium. ${ }^{26,} 33$ Quantitative monosaccharide composition and 267 methylation (monosaccharide linkage) analyses indicated that the EPS fraction of low MM 268 (peak 2) produced by both bifidobacteria was an HePS mainly composed of rhamnose, 
269 galactose and glucose (Table 1). Traces of mannose, xylose, N-acetyl-glucosamine and 270 glucuronic acid were also found (data not shown). FT-IR spectra of these HePS had similar 271 patterns and showed absorption bands characteristic of neutral polysaccharides (data not 272 shown). Thus, from our results it is not clear at this point whether the proteins that 273 appeared associated with the peak of low MM (peak 2) detected by SEC-MALLS were 274 cellular contaminants or take part of the structure of the polymer; in any case, slight 275 differences in monosaccharide proportions and linkage types were found between polymers of peak 2 from parental and derivative strains. Thus, a slightly lower proportion of glucose and galactose was obtained in the polymer of B. longum IPLA B667dCo as compared to the polymer of the parental strain (Table 1); this feature was consistent with the lower proportion of residues $\rightarrow 4)-\mathrm{Glc} p-(1 \rightarrow$ and $\rightarrow 3)-\mathrm{Gal} p-(1 \rightarrow$ in the EPS fraction of

280 the derivative strain (Table 2). Relating to this, we have recently reported variations in MM and monosaccharide ratios between polymers synthesized by a bile resistant $B$. animalis and its parental $\operatorname{strain}^{14}$ as well as slight variations in monosaccharide linkage proportions between EPS produced by a L. delbrueckii subsp. lactis strain and its bile resistant derivative. ${ }^{15}$ All these findings suggest that adaptation to bile could promote changes in the composition and physico-chemical characteristics of EPS.

The carbohydrate content of the high MM peak (peak 1) was similar in EPS from both B. longum NB667 and IPLA B667dCo strains (Table 1). It consisted of a HePS formed by galactose and glucose in molar ratios of 1:1.8, which presented similar 289 proportions of sugar linkage types in both microorganisms (Table 2). Traces of mannose 290 were also found. To have a more precise characterization of this high MM fraction and to 291 determine possible differences involving non-carbohydrate molecules, the FT-IR spectra in 292 the region 3,750-400 $\mathrm{cm}^{-1}$ was obtained and analyzed (Figure 3). The intensity of bands 293 around $3,400,1,400$, and $1,060 \mathrm{~cm}^{-1}$ is due to the hydroxyl stretching vibration of the 
294 polysaccharides, and was similar in the peaks of both polymers. The absorption band at $2952,927 \mathrm{~cm}^{-1}$ corresponds to methyl groups and it was also present in this polymer fraction of 296 both microorganisms; since no monosaccharides containing methyl groups were found in 297 this peak, the presence of this FT-IR band may be associated to other non-carbohydrate 298 structures taking part of the polymer. The region between 1,860 and $1,660 \mathrm{~cm}^{-1}$ revealed 299 the presence of carbonyl groups. In this region, some differences were evidenced between 300 the high MM EPS fractions synthesized by the parental and the cholate-resistant strains. 301 Thus, in EPS NB667 a weak absorption band appeared at $1,727 \mathrm{~cm}^{-1}$ whereas a 302 considerably more intense band appeared at $1,685 \mathrm{~cm}^{-1}$ in the EPS fraction of the cholate303 resistant strain IPLA B667dCo. Some differences were also found at 1,209 and 1,131 cm

304 between fractions of both strains. Moreover, DOSY spectra also corroborated a higher 305 heterogeneity in the peak 1 from EPS IPLA B667dCo than in the peak 1 from EPS NB667 306 (data not shown). These results indicated that the fractions of high MM found in EPS 307 polymers from the strains $B$. longum NB667 and IPLA B667dCo were equal in 308 monosaccharide content, but presented some differences in the infrared spectra regarding 309 compounds other than carbohydrates that could also be associated to the polymers. The 310 presence of these non-carbohydrate structures may be the reason for the low 311 monosaccharide recovery obtained after the acid hydrolysis of the high MM fraction; this

312 fact and mismatches between the hydrodynamic volume and the true Mw may be 313 contributing to the discrepancies observed between the apparent MM and Mw obtained for

314 peak 1 using different techniques. The elucidation of the chemical nature of these non315 carbohydrate residues and the structure of the high MM fraction present in the EPS 316 synthesized by the parental and the cholate derivative B. longum strains should take 317 advantage from using nuclear magnetic resonance spectroscopy and mass spectrometry 318 techniques and constitutes a research challenge for the near future. Thus, it seems that the 
319 adaptation to bile salts could not only induce the synthesis of EPS ${ }^{13}$, but also produce

320 modifications in the physico-chemical characteristics of the synthesized polymers.

EPS and cell wall polysaccharides described so far in the species B. longum are

322 HePS, which seems to be a common feature in the genus Bifidobacterium. ${ }^{26-28,30-33}$

323 Galactose is present in all polymers of the species B. longum currently known, being the

324 major component of most of them, ${ }^{26,28,30-32}$ as it was also the case of the EPS produced

325 by our strains B. longum NB667 and IPLA B667dCo. Indeed, in the peak of low MM

326 galactose was the most abundant monosaccharide.

\section{Fermentation of crude EPS fractions by the human intestinal microbiota}

The bifidogenic effect of EPS purified from intestinal bifidobacteria isolated

330 from healthy adults, as well as their ability to be fermented by the human intestinal

331 microbiota has recently been demonstrated in our group. ${ }^{12,17}$ In the same way, we have

332 used in the current work the crude EPS polymers isolated from the strain NB667 from

333 infant origin, and from the strain IPLA B667dCo, adapted to cholate, in order to know if

334 they can also be fermented by the intestinal microbiota and whether the physico-

335 chemical changes promoted by the acquisition of resistance to cholate could modify the

336 general fermentation pattern of B. longum EPS preparations.

337 The increase of total SCFA after 5 days of incubation in fecal cultures was

338 significantly $(\mathrm{P}<0.05)$ more pronounced in the presence of EPS than in the control

339 cultures without any carbon source added, indicating that the EPS from $B$. longum were

340 fermented by the intestinal bacteria (Table 3). Acetic acid was the most abundant

341 SCFA, followed by propionic and butyric acids. Molar proportions of acetic and

342 propionic acids decreased and increased, respectively, during incubation in the presence 
343 of EPS preparations of both strains, whereas proportions of butyric acid did not suffer

344 noticeable variations. This fermentation pattern coincided with that previously observed

345 by us for other EPS preparations from B. longum strains of human intestinal origin. ${ }^{12}$ As

346 a consequence of the SCFA production pattern just indicated, the acetic acid to

347 propionic acid ratio decreased during incubation in the presence of EPS from all

348 microorganisms tested in the present work and in previous studies. Interestingly,

349 cultures with EPS NB667 and IPLA B667dCo displayed slightly more pronounced

350 decreases and lower final values for the acetic acid to propionic acid ratio than cultures

351 with EPS from other bifidobacteria of adult intestinal origin previously tested by us,

352 including several isolates of $B$. longum species. ${ }^{12}$ In spite of this, no significant

353 differences were evidenced in the present work on the levels of total SCFA, acetic,

354 propionic and butyric acids formed during incubation between fecal cultures with EPS

355 preparations of the strains B. longum NB667 and IPLA B667dCo (Table 3). The

356 reduction in the acetic acid to propionic acid ratio has been proposed as a possible

357 indicator of the hypolipidemic effect of prebiotics, ${ }^{34}$ leading to a decrease of lipid levels

358 in blood.

359 The high inter-individual difference among fecal cultures of the different 360 donors precluded the statistical analysis; then, qPCR counts of fecal cultures of each

361 individual were considered separately. A moderate stimulatory effect on intestinal

362 Bifidobacterium populations as compared to the control (without carbohydrate added),

363 was obtained for EPS crude preparations of both B. longum strains in the fecal cultures

364 of only one individual (donor 3) out of the three analyzed (Figure 4). This behavior was

365 similar to that previously reported with EPS from other bifidobacteria of adult intestinal

366 origin. $^{12}$ No remarkable differences were found between the crude EPS preparations

367 from the parental and the cholate-resistant strains, as it can be expected from minor 
variations in the carbohydrate composition encountered between the polymers of both

369 microorganisms.

370

Several surface macromolecules of Gram-positive bacteria, among which are

372 glycosylated structures and EPS, could act as signal molecules interacting with eukaryotic

373 cells of the host. ${ }^{35}$ Thus, variations in composition, as well as in proportions, between the

374 different monosaccharides and linkage types could modify the structural and functional

375 characteristics of the polymers and hence the possible influence in the cross-talk mechanisms of the producing bacteria with the host. In this way, we have reported a notable increase in hydrophobicity as well as enhanced in vitro adhesion to human intestinal mucus of the cholate-resistant strain B. longum IPLA B667dCo with respect to the parental sensitive strain B. longum NB667. ${ }^{36}$

In the current work we have corroborated, by using two strains of the species

B. longum as a model of study, that the acquisition of bile salt resistance promoted changes in the physico-chemical characteristics of EPS. Thus, we suggest that these modifications in EPS production may be a mechanism of response to bile stress although this hypothesis, as well as the ecological relevance of these changes, deserves future confirmation. On the other hand, differences in composition and glycosidic linkages of the polymer fractions produced by parental and cholate-resistant strains were not affecting their capability to be fermented by the intestinal microbiota. To the best of our knowledge this is the first report on changes promoted by the acquisition of a stable bile-resistance phenotype in the characteristics of EPS produced by microorganisms of the species B. longum. 


\section{LITERATURE CITED:}

(1) Harmsen, H.J.; Wideboer-Veloo, A.C; Raangs, G.C.; Wagendorp, A.A.; Klijn, N.; Bindels, J.G.; Welling, G.W. Analysis of intestinal flora development in breast-fed and formula-fed infants by using molecular identification and detection methods. J. Pediatr. Gastroenterol. Nutr. 2000, 30, 61-67. dependent and culture-independent qualitative analysis of probiotic products claimed to contain bifidobacteria. Int. J. Food Microbiol. 2005, 102, 221-230. environmental challenges? Mechanisms involved and physiological consequences. Genes Nutr. 2011, 6, 307-318.

405

(4) Gueimonde, M.; Margolles, A.; de los Reyes-Gavilán, C.G.; Salminen, S. Competitive exclusion of enteropathogens from human intestinal mucus by Bifidobacterium strains with acquired resistance to bile-a preliminary study. Int. J. Food Microbiol. 2007, 113, 228-232. Gavilán, C.G. Effect of the adaptation to high bile salts concentrations on glycosidic activity, survival at low $\mathrm{pH}$ and cross-resistance to bile salts in Bifidobacterium. Int. J. Food Microbiol. 2004, 94, 79-86. bile salt resistance promotes antibiotic susceptibility changes in Bifidobacterium. 
(7) Ruas-Madiedo, P.; Hernández-Barranco, A.M.; Margolles, A.; de los

Reyes-Gavilán, C.G. A bile salt-resistant derivative of Bifidobacterium animalis has an altered fermentation pattern when grown on glucose and maltose. Appl. Environ. Microbiol. 2005, 71, 6564-6570.

(8) Abraham, A.G.; Medrano, M.; Piermaria, J.A.; Mozzi, F. Novel applications of polysaccharides from lactic acid bacteria: a focus on kefiran. In Food Hydrocolloids: characteristics, properties and structures. Hollingworth, C.S. Ed.; Nova Publishers: New York, 2010; 253-271. Exopolysaccharides of lactic acid bacteria: a review. J. Food Sci. Technol. 2009, $46,1-11$.

(10) Ruas-Madiedo, P.; Salazar, N.; de los Reyes-Gavilan, C.G. Functionality of exopolysaccharides produced by lactic acid bacteria. In Molecular aspects of lactic acid bacteria for traditional and new applications. Mayo, B.; López, P.; Pérez-Martínez, G., Eds.; Research Signpost: Trivandrum, Kerala, India, 2008; 137-166.

(11) Ruas-Madiedo, P.; Moreno, J.A.; Salazar, N.; Delgado, S.; Mayo, B.; Margolles, A.; de los Reyes-Gavilán, C.G. Screening of exopolysaccharideproducing Lactobacillus and Bifidobacterium strains isolated from the human intestinal microbiota. Appl. Environ. Microbiol. 2007, 73, 4385-4388.

(12) Salazar, N.; Gueimonde, M.; Hernández-Barranco, A.M.; Ruas-Madiedo, P.; de los Reyes-Gavilán, C.G. Exopolysaccharides produced by intestinal Bifidobacterium strains act as fermentable substrates for human intestinal bacteria. Appl. Environ. Microbiol. 2008, 74, 4737-4745. 
C.G.; Margolles, A. Bile affects the synthesis of exopolysaccharides by Bifidobacterium animalis. Appl. Environ. Microbiol. 2009, 75, 1204-1207.

(14) Ruas-Madiedo, P.; Medrano, M.; Salazar, N.; de los Reyes-Gavilán, C.G.; Pérez, P.F.; Abraham, A.G. Exopolysaccharides produced by Lactobacillus and Bifidobacterium strains abrogate in vitro the cytotoxic effect of bacterial toxins on eukaryotic cells. J. Appl. Microbiol. 2010, 109, 2079-2086. (15) Burns, P.; Vinderola, G.; Reinheimer, J.; Cuesta, I.; de los ReyesGavilán, C.G.; Ruas-Madiedo. P. Technological characterization and survival of the exopolysaccharide-producing strain Lactobacillus delbrueckii subsp. lactis juices. J. Dairy Res. 2011, 78, 357-364. 193 and its bile-resistant derivative 193+ in simulated gastric and intestinal

(16) Ruas-Madiedo, P.; Gueimonde, M.; Margolles, A.; de los Reyes-Gavilán, C.G.; Salminen, S. Exopolysaccharides produced by probiotic strains modify the adhesion of probiotics and enteropathogens to human intestinal mucus. J. Food Prot. 2006, 69, 2011-2015.

(17) Salazar, N.; Ruas-Madiedo, P.; Kolida, S.; Collins, M.; Rastall, R.; Gibson, G.; de los Reyes-Gavilán, C.G. Exopolysaccharides produced by Bifidobacterium longum IPLA E44 and Bifidobacterium animalis subsp. lactis IPLA R1 modify the composition and metabolic activity of human faecal microbiota in pH-controlled batch cultures. Int. J. Food Microbiol. 2009, 135, $260-267$.

(18) Stilbs, P. Molecular self-diffusion coefficients in Fourier transform nuclear magnetic resonance spectrometric analysis of complex mixtures. Anal. Chem. 1981, 53, 2135-2137. 
(19) Leal, J.A.; Giménez-Abián, M.I.; Canales, Á.; Jiménez-Barbero, J.;

Bernabé, M.; Prieto, A. Cell wall polysaccharides isolated from the fungus Neotestudina rosatii, one of the etiologic agents of mycetoma in man. Glycoconj. J. 2009, 26, 1047-1054.

(20) Dubois, M.; Gilles, K.A.; Hamilton, J.K.; Rebers, P.A.; Smith, F. Colorimetric method for the determination of sugar and related substances. Anal. Chem. 1956, 28, 350-356.

(21) Laine, R.A.; Esselman, W.J.; Sweeley, C.C. Gas-liquid chromatography of carbohydrates. Meth. Enzymol. 1972, 28, 159-167.

(22) Ciucanu, I.; Kerek, F. A simple and rapid method for the permethylation of carbohydrates. Carbohydr. Res. 1984, 13, 209-217. elucidation of a cell wall fungal polysaccharide isolated from Ustilaginoidea virens, a pathogenic fungus of Oriza sativa and Zea mays. Carbohydr. Res. 2008, 343, 2980-2984.

(24) Price, W.J. Sample handling techniques, In Laboratory methods in infrared spectroscopy. R.G.J. Miller, R.G.J.; Stacc, B.C., Eds.; Heyden and Son: London, England, 1972; 97-128. quantitative PCR procedure for quantification of bifidobacteria in human fecal samples. Appl. Environ. Microbiol. 2004, 70, 4165-4169. and characterization of exocellular polysaccharides produced by Bifidobacterium longum. Appl. Microbiol. Biotechnol. 1995, 43, 995-1000. 
Reyes-Gavilán, C.G.; Ruas-Madiedo, P. Production of exopolysaccharides by Lactobacillus and Bifidobacterium strains from human origin and metabolic activity of the producing bacteria in milk. J. Dairy Sci. 2009, 92, 4158-4168.

(28) Tone-Shimokawa, Y.; Toida, T.; Kawashima, T. Isolation and structural analysis of polysaccharide containing galactofuranose from the cell walls of Bifidobacterium infantis. J. Bacteriol. 1996, 178, 317-320. (29) Mozzi, F.; Vaningelgem, F.; Hébert, E.M.; van der Meulen, R.; FoulquiéMoreno, M.R.; Font de Valdéz, G.; De Vusyt, L. Diversity of heteropolysaccharide-producing lactic acid bacterium strains and their biopolymers. Appl. Environ. Microbiol. 2006, 72, 4431-4435. Bifidobacterium longum YIT4028. Carbohydr. Res. 1995, 274, 245-249. Hoover, D.G. Exopolysaccharide production by Bifidobacterium longum BB-79. J. Appl. Bacteriol. 1995, 78, 463-468. produced by Bifidobacterium longum JBL05. Carbohydr. Polym. 2009, 77, 351357.

(33) Xu, R.; Shen, Q; Ding, X.; Gao, W; Li, P. Chemical characterization and antioxidant activity of an exopolysaccharide fraction isolated from Bifidobacterium animalis RH. Eur. Food Res. Technol. 2011, 232, 231-240.

(34) Delzenne, N.M.; Kok, N. Effects of fructan-type prebiotics on lipid metabolism. Am. J. Clin. Nutr. 2001, 73, 456S-458S. 
(35) Lebeer, S.; Vanderleyden, J.; de Keersmaecker, S.C.J. Host interactions of probiotic bacterial surface molecule: comparison with commensals and pathogens. Nat. Rev. Microbiol. 2010, 8, 171-184.

(36) Gueimonde, M.; Noriega, L.; Margolles, A.; de los Reyes-Gavilán, C.G.; Salminen, S. Ability of Bifidobacterium strains with acquired resistance to bile to adhere to human intestinal mucus. Int. J. Food Microbiol. 2005, 101, 341-346. 


\section{FIGURE CAPTIONS}

Figure 1. Size exclusion chromatography (SEC) analysis of the EPS crude fractions produced by the strains Bifidobacterium longum NB667 and IPLA B667dCo. The EPS were dissolved in $0.1 \mathrm{M} \mathrm{NaNO}_{3}\left(5 \mathrm{mg} \mathrm{ml}^{-1}\right)$ and separation was carried out in a HPLC system (Alliance 2690 module injector, Waters, Milford, MA, USA) using two TSKGel columns (Sigma-Supelco) placed in series $\left(\mathrm{G} 3000 \mathrm{PW}_{\mathrm{XL}}+\mathrm{G} 5000 \mathrm{PW}_{\mathrm{XL}}\right.$ protected with a TSK-Gel guard column) at $40^{\circ} \mathrm{C}$ using $0.1 \mathrm{M} \mathrm{NaNO}_{3}$ as mobile phase at flow rate $0.45 \mathrm{ml} \mathrm{min}^{-1}$. After separation, three detectors placed in series were used: the refraction index detector (Waters RI, blue line) was used for detection of different molecules, the photodiode array (Waters PDA, green line) set at $280 \mathrm{~nm}$ was used to identify the presence of proteins, and the multiangle laser light scattering (MALLS) detector Dawn Heleos II (Wyatt Europe GmbH, Dembach, Germany) having eighteen angles of detection (detector $11=$ angle $90^{\circ}$, red line) was used to study the molar mass distribution of the EPS fractions. The ASTRA software (Wyatt Europe) was used to analyse the scattering data using the Random Coil adjust-model.

Figure 2. Preparative size exclusion chromatography (pre-SEC) of the EPS crude polymers synthesised by the strains Bifidobacterium longum NB667 and IPLA B667dCo. Carbohydrates in eluted fractions were measured by the phenol-sulfuric acid method. Arrows indicate fractions collected from each elution peak. EPS NB667 (peak 1: fractions 28-48, peak 2: fractions 62-84); EPS IPLA B667dCo (peak 1: fractions 3044, peak 2: fractions 60-85).

Figure 3. Fourier-transform infrared spectra of the peak of high MM purified by preparative-SEC in Sepharose CL6B from crude EPS polymers of the strains 
Bifidobacterium longum NB667 (upper line) and B. longum IPLA B667dCo (bottom line). $\% \mathrm{~T}=$ percentage of transmittance. The dotted rectangles indicate areas of the spectrum displaying differences between parental and cholate resistant strains.

Figure 4. Increase, with respect to time zero, of Bifidobacterium counts measured by qPCR in fecal slurry cultures from three human adult donors in the absence of external carbohydrate added (control, white bars) and using crude EPS isolated from the strains Bifidobacterium longum NB667 (gray bars) and B. longum IPLA B667dCo (black bars) as carbon sources after 1 day (a) and 5 days (b) of incubation. Initial Bifidobacterium counts were as follows: $9.64 \pm 0.10 \log$ cells $\mathrm{g}^{-1}$ for donor $1,10.65 \pm 0.12 \log$ cells $\mathrm{g}^{-1}$ for donor 2, and $9.93 \pm 0.08 \log$ cells $\mathrm{g}^{-1}$ for donor 3. Vertical lines indicate standard deviation. 
Table 1. Physico-chemical characteristics of the crude EPS polymers produced by the strains Bifidobacterium longum NB667 and IPLA B667dCo. The weight average molar mass $\left(\mathrm{M}_{\mathrm{w}}, \mathrm{g} \mathrm{mol}^{-1}\right)$, weight radius of gyration $\left(\mathrm{R}_{\mathrm{w}}, \mathrm{nm}\right)$ as well as the coefficient $v\left(\log \mathrm{R}_{\mathrm{w}} / \log \mathrm{M}_{\mathrm{w}}\right)$ were determined by SEC-MALLS (see Figure 1). The crude EPS polymers were fractionated by preparative SEC (see Figure 2) and the apparent average molar mass $(\mathrm{MM})\left(\mathrm{g} \mathrm{mol}^{-1}\right)$ of the fractions (peaks) as well as their monosaccharide composition were determined by phenol-sulfuric method using commercial standards, and GC-FID, respectively. Glc: glucose, Gal: galactose, Rha: rhamnose.

\section{EPS NB667}

\begin{tabular}{|c|c|c|c|c|c|c|}
\hline & & \\
\hline & Crude EPS & Peak 1 & Peak 2 & Crude EPS & Peak 1 & Peak 2 \\
\hline $\mathrm{M}_{\mathrm{w}}$ & - & $5.5 \times 10^{5}$ & $7.2 \times 10^{3}$ & - & $7.8 \times 10^{5}$ & $7.6 \times 10^{3}$ \\
\hline $\mathrm{R}_{\mathrm{w}}(\mathrm{nm})$ & - & 67.8 & 22.9 & - & 69.8 & 24.4 \\
\hline$v\left(\log \mathrm{R}_{\mathrm{w}} / \log \mathrm{M}_{\mathrm{w}}\right)$ & - & 0.35 & 1.52 & - & 0.31 & 1.18 \\
\hline $\begin{array}{l}\text { Apparent MM and } \\
\text { distribution }(\%)\end{array}$ & - & $\begin{array}{r}4.3 \times 10^{6} \\
(49 \%)\end{array}$ & $\begin{array}{r}4.3 \times 10^{3} \\
(51 \%)\end{array}$ & - & $\begin{array}{c}5.4 \times 10^{6} \\
(41 \%)\end{array}$ & $\begin{array}{r}4.5 \times 10^{3} \\
(59 \%)\end{array}$ \\
\hline \multicolumn{7}{|c|}{ Monosaccharide ratio } \\
\hline Glc & 1.0 & 1.8 & 1.2 & 1.2 & 1.8 & 1.0 \\
\hline Gal & 2.2 & 1.0 & 1.8 & 2.3 & 1.0 & 1.7 \\
\hline Rha & 1.0 & 0.0 & 1.0 & 1.0 & 0.0 & 1.0 \\
\hline
\end{tabular}


Table 2. Linkage types (ratios) deduced for EPS produced by the strains Bifidobacterium longum NB667 and B. longum IPLA-B667dCo. Glc: glucose, Gal: galactose, Rha: rhamnose, Hex: hexose, $p$ : pyranose ring conformation, $f$ : furanose ring conformation.

\begin{tabular}{|c|c|c|c|c|}
\hline \multirow{2}{*}{ Linkage } & \multicolumn{2}{|c|}{ EPS NB667 } & \multicolumn{2}{|c|}{ EPS IPLA-B667dCo } \\
\hline & Peak 1 & Peak 2 & Peak 1 & Peak 2 \\
\hline Glcp- $(1 \rightarrow$ & 0.5 & & 0.5 & \\
\hline$\rightarrow 2,4)$-Glcp-( $1 \rightarrow$ & 1.0 & & 1.0 & \\
\hline$\rightarrow 4)-G a l p-(1 \rightarrow$ & 1.0 & 0.2 & 1.0 & 0.2 \\
\hline$\rightarrow 4)$-Glcp-(1 $\rightarrow$ & 0.7 & 1.2 & 0.7 & 0.7 \\
\hline Hexp-(1) & & 0.1 & & 0.3 \\
\hline Galp-(1) & & 0.9 & & 0.9 \\
\hline$\rightarrow 2)$-Rhap-(1 $\rightarrow$ & & 1.0 & & 1.0 \\
\hline$\rightarrow 3)$-Galf $f(1 \rightarrow$ & & 0.1 & & 0.1 \\
\hline$\rightarrow 3)-G a l p-(1 \rightarrow$ & & 0.7 & & 0.6 \\
\hline$\rightarrow 6)-$ Нех $p-(1 \rightarrow$ & & 0.2 & & 0.2 \\
\hline$\rightarrow 3,6)$-Galp-(1 $\rightarrow$ & & 1.0 & & 1.0 \\
\hline
\end{tabular}


Table 3. Molar concentrations and proportion of the three major SCFA in fecal cultures from three adult healthy donors without carbohydrate added (control) and using isolated crude EPS preparations from Bifidobacterium longum NB667 and B. longum IPLA-B667dCo as carbon sources. The differences among the three "carbon source" groups in each sampling point were tested by means of independent one-way ANOVA. The means that do not share a common superscript are significantly different $(\mathrm{p}<0.05)$ accordingly to the mean comparison test LSD.

\begin{tabular}{|c|c|c|c|c|c|c|c|c|c|}
\hline \multirow{2}{*}{$\begin{array}{l}\text { Time } \\
\text { (days) }\end{array}$} & \multirow[t]{2}{*}{ Carbon source } & \multicolumn{4}{|c|}{ Concentration $\pm \mathrm{SD}(\mathrm{mM})$} & \multicolumn{4}{|c|}{ Proportion \pm SD $(\%)$} \\
\hline & & Total acid & Acetate & Propionate & Butyrate & Acetate & Propionate & Butyrate & $\mathrm{A} / \mathrm{P}$ ratio \\
\hline 0 & Control & $6.27 \pm 2.68$ & $4.10 \pm 1.86$ & $0.96 \pm 0.40$ & $1.22 \pm 0.42$ & $64.77 \pm 1.82$ & $15.27 \pm 1.23$ & $19.95 \pm 2.15$ & $4.26 \pm 0.35$ \\
\hline \multirow{3}{*}{1} & Control & $13.72 \pm 1.13$ & $8.42 \pm 0.47$ & $2.27 \pm 0.26$ & $3.03 \pm 0.55$ & $61.44 \pm 1.93$ & $16.59 \pm 1.71$ & $21.97 \pm 2.15$ & $3.73 \pm 0.41$ \\
\hline & EPS NB667 & $14.95 \pm 4.02$ & $9.12 \pm 2.48$ & $2.80 \pm 0.78$ & $3.03 \pm 0.88$ & $61.08 \pm 3.14$ & $18.74 \pm 2.63$ & $20.18 \pm 0.69$ & $3.31 \pm 0.59$ \\
\hline & EPS IPLA-B667dCo & $14.83 \pm 0.41$ & $8.88 \pm 0.43$ & $2.80 \pm 0.36$ & $3.15 \pm 0.46$ & $59.96 \pm 4.15$ & $18.87 \pm 2.24$ & $21.17 \pm 2.54$ & $3.22 \pm 0.61$ \\
\hline \multirow{3}{*}{5} & Control & $12.33 \pm 1.70^{\mathrm{a}}$ & $8.16 \pm 1.33^{\mathrm{a}}$ & $1.90 \pm 0.67^{\mathrm{a}}$ & $2.27 \pm 0.79$ & $66.34 \pm 7.55$ & $15.10 \pm 3.51^{\mathrm{a}}$ & $18.55 \pm 6.32$ & $4.58 \pm 1.27^{\mathrm{b}}$ \\
\hline & EPS NB667 & $18.65 \pm 2.66^{\mathrm{b}}$ & $10.23 \pm 0.63^{b}$ & $5.11 \pm 1.22^{\mathrm{b}}$ & $3.31 \pm 0.82$ & $55.33 \pm 4.98$ & $21.12 \pm 2.92^{\mathrm{b}}$ & $17.55 \pm 2.07$ & $2.07 \pm 0.43^{\mathrm{a}}$ \\
\hline & EPS IPLA-B667dCo & $20.71 \pm 4.17^{\mathrm{b}}$ & $11.16 \pm 1.26^{\mathrm{b}}$ & $5.82 \pm 1.72^{\mathrm{b}}$ & $3.72 \pm 1.24$ & $54.60 \pm 5.58$ & $27.74 \pm 3.20^{\mathrm{b}}$ & $17.65 \pm 2.47$ & $2.00 \pm 0.46^{\mathrm{a}}$ \\
\hline
\end{tabular}


Fig. 1
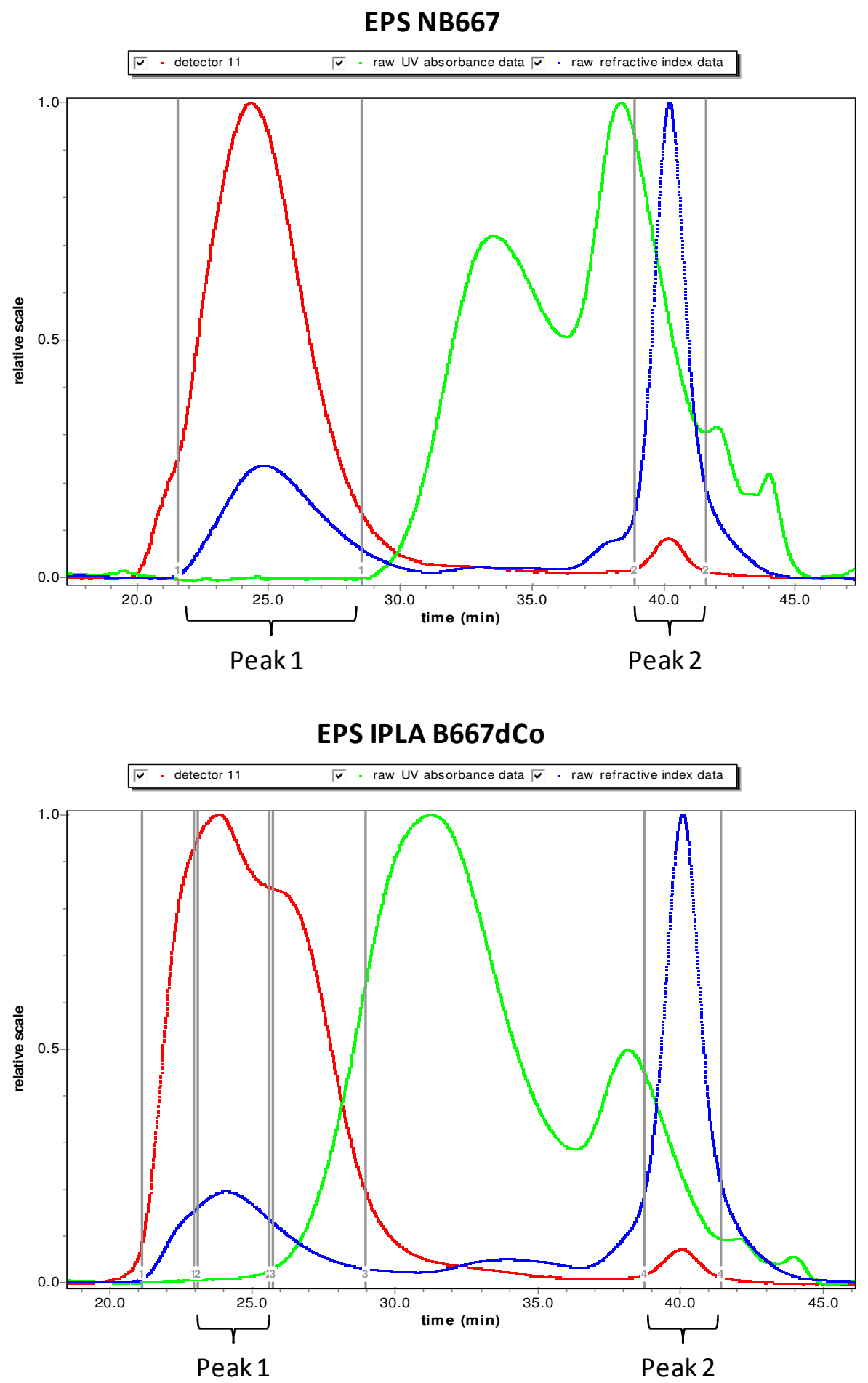
Fig. 2
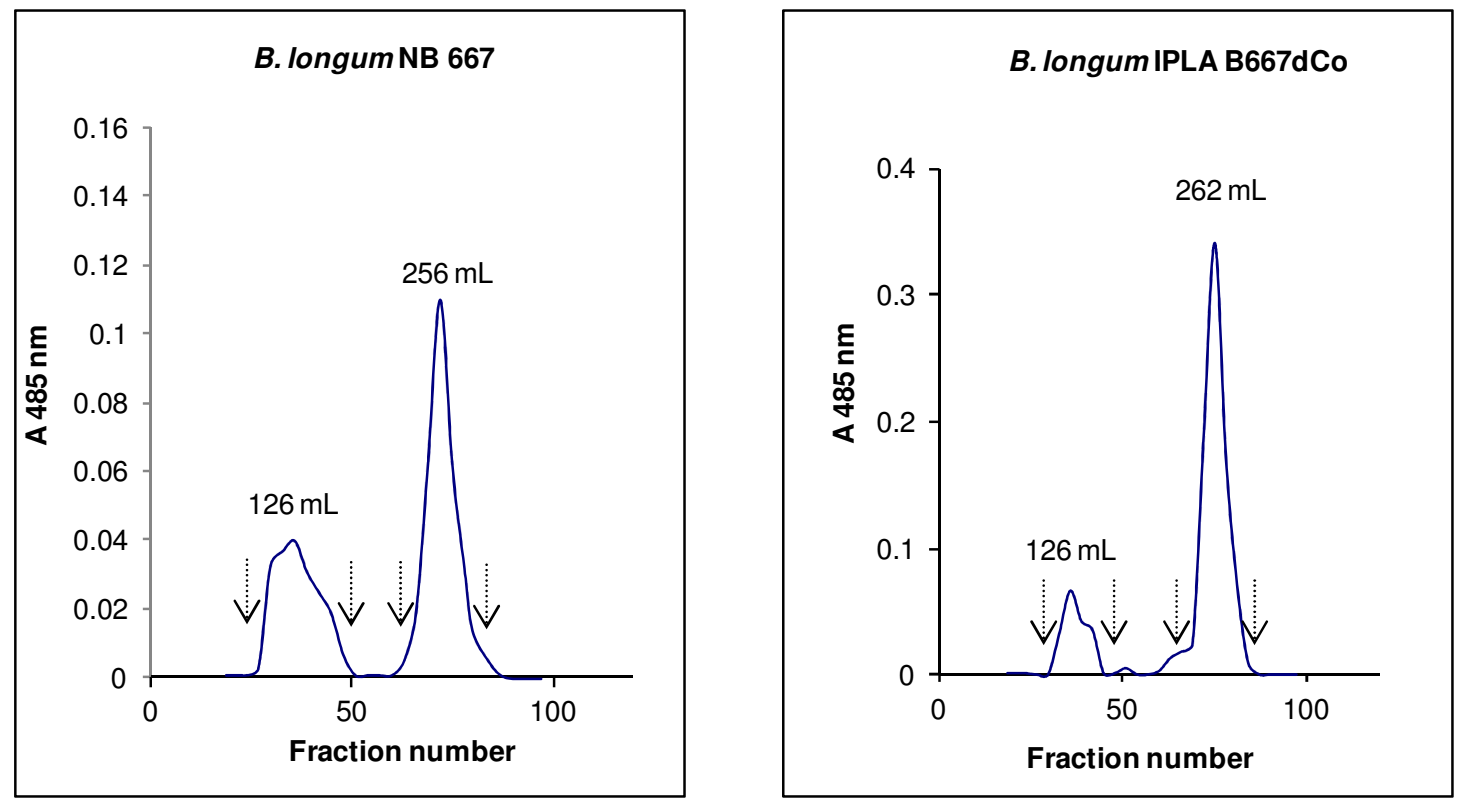


\section{Fig. 3}

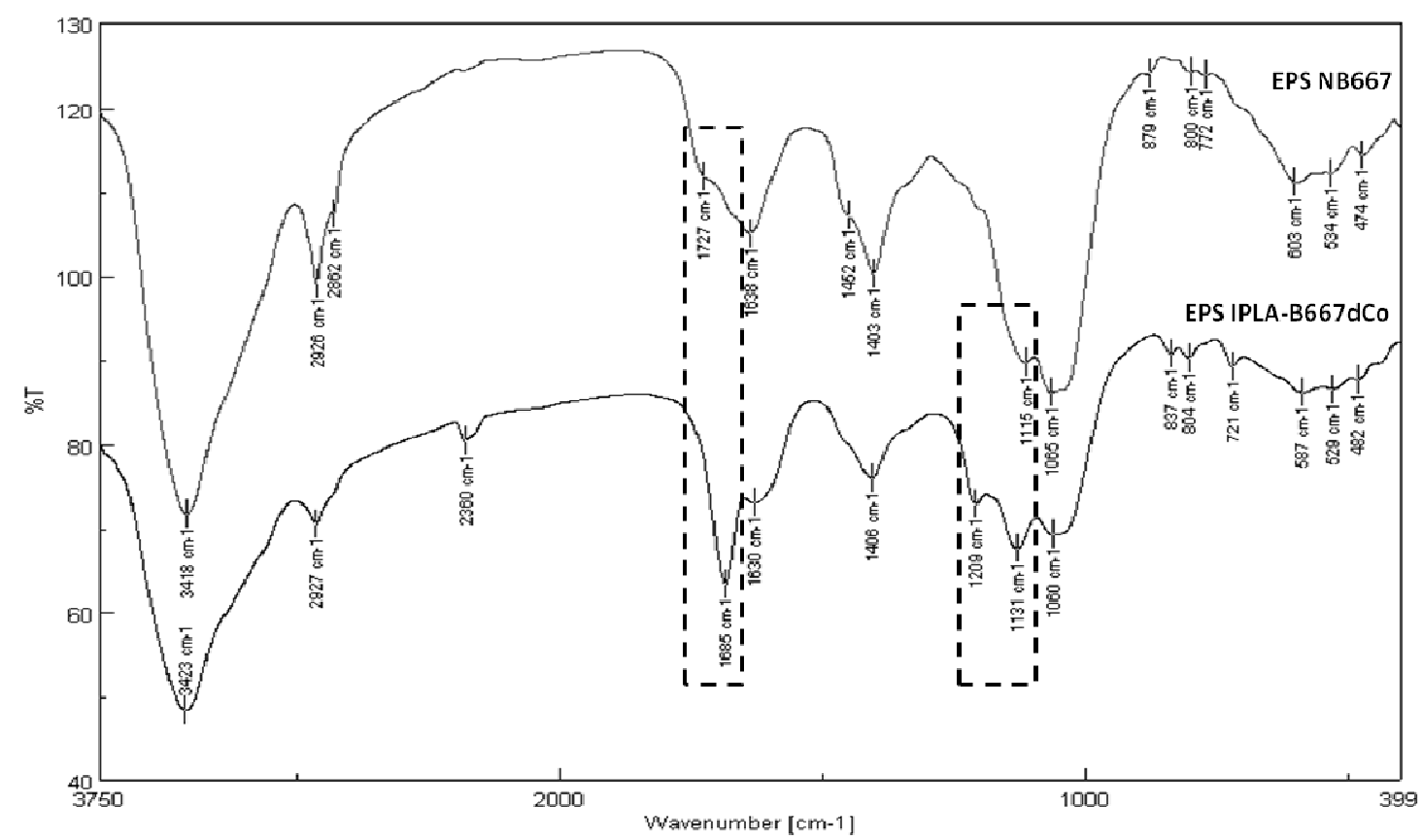


Fig. 4
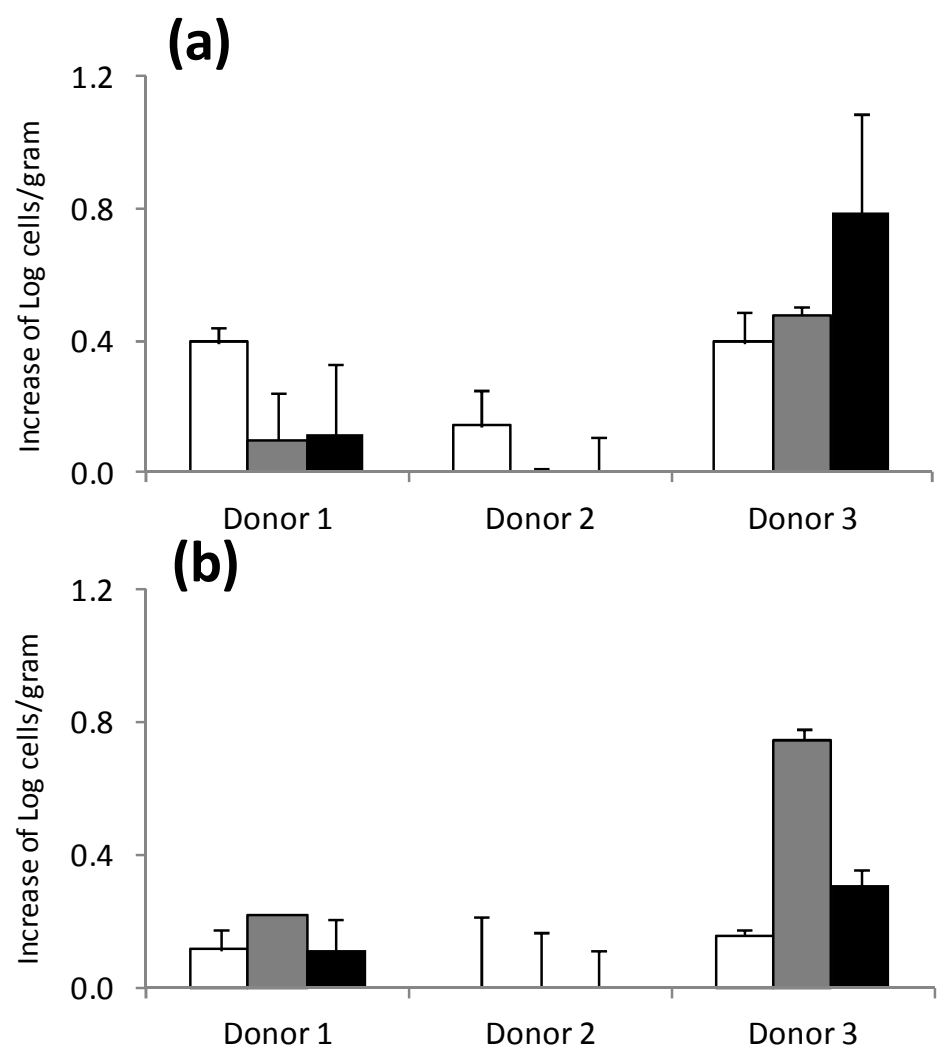


\section{Figure for the Table of Contents}
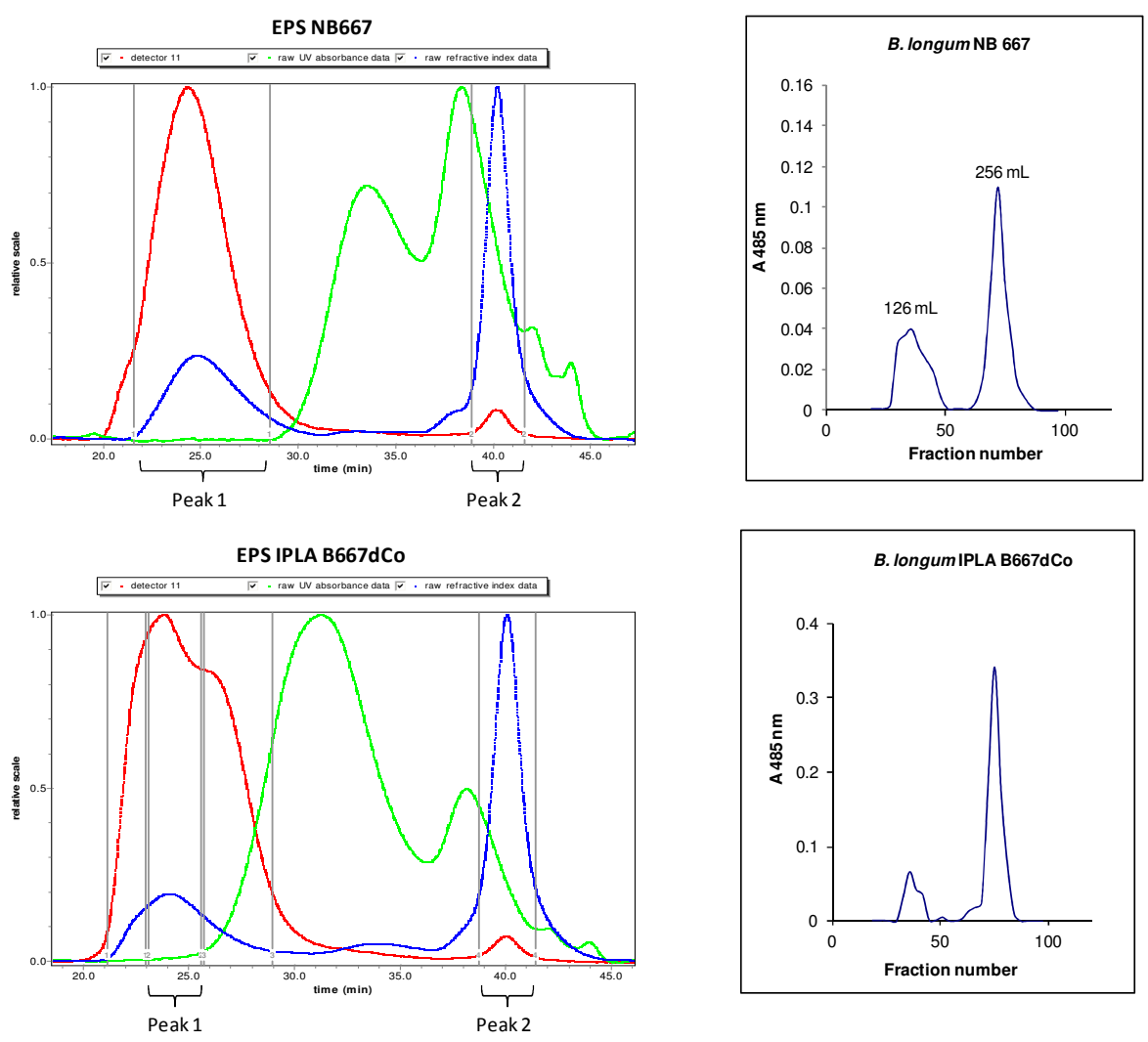